\title{
Possible associations between ascites and vascular invasion in patients with hepatocellular carcinoma
}

\author{
Ling Deng ${ }^{1}$ • Hong-Gui Qin ${ }^{2}$ - Chun Yang ${ }^{1}$ J Jian-Hong Zhong ${ }^{2}$
}

Received: 16 April 2015 / Accepted: 28 April 2015 /Published online: 3 May 2015

(C) International Society of Oncology and BioMarkers (ISOBM) 2015

Hepatocellular carcinoma (HCC) is a common malignancy, and its incidence is expected to increase in many countries in coming decades [1]. The main risk factors for the development of HCC are chronic infection with hepatitis $\mathrm{B}$ virus (HBV) or hepatitis $\mathrm{C}$ virus $(\mathrm{HCV})$ as well as alcoholic cirrhosis [2].

Though prognosis for patients with $\mathrm{HCC}$ is generally poor, hepatic resection (HR) can be an effective curative treatment, and its indications have been expanding in recent years [3, 4]. HR can be reasonably safe and effective even for patients with 2-3 tumors with maximum diameter $>3 \mathrm{~cm}$, patients with $>3$ tumors regardless of size, and patients with macrovascular invasion $[5,6]$. However, the recurrence rate of HCC is as high as $74 \%$ for patients with intermediate and advanced HCC after HR [7]. Many factors influence patient prognosis and risk of HCC recurrence after HR; these factors can be due to the characteristics of the HCC itself as well as to the characteristics of the remnant liver. Identifying these risk factors is important for decreasing the recurrence rate and improving overall survival.

A recent issue of Tumour Biology contains an interesting retrospective study by Chen and coworkers based on 336

Ling Deng, Hong-Gui Qin and Chun Yang contributed equally to this work.

Jian-Hong Zhong

zhongjianhong66@163.com

1 Department of Experimental Pathology, Affiliated Tumor Hospital of Guangxi Medical University, Nanning 530021, People's Republic of China

2 Department of Surgical Oncology, Affiliated Tumor Hospital of Guangxi Medical University, He Di Rd. \#71, Nanning 530021, People's Republic of China patients with HBV-related cirrhotic HCC, of which histopathology data were available for 153 [8]. Multivariate analyses revealed that factors predicting macrovascular invasion $(n=$ 336) included tumor size $>5 \mathrm{~cm}$, metastases, presence of ascites, and $\alpha$-fetoprotein $>1000 \mathrm{ng} / \mathrm{mL}$. Factors predicting microvascular invasion, based on subgroup analysis of 128 patients, were high-grade tumor differentiation, presence of ascites, and $\alpha$-fetoprotein $>1000 \mathrm{ng} / \mathrm{mL}$. The presence of ascites and $\alpha$-fetoprotein $>1000 \mathrm{ng} / \mathrm{mL}$ were both risk factors of macro- and microvascular invasion.

While this study provides some important information about risk factors of recurrence rate for patients with $\mathrm{HCC}$, it fails to examine any of the most clinically relevant and valuable outcomes of recurrence rate and overall or disease-free survival. It is essential to compare survival curves between patients with and without macro- or microvascular invasion, ascites, or $\alpha$-fetoprotein $>1000 \mathrm{ng} / \mathrm{mL}$ in order to know whether these characteristics, which statistically may qualify as "risk factors," actually predict risk of clinically relevant outcomes of recurrence and survival. This is important not just for clinical relevance but also for the credibility of the analysis: if the risk factors identified by Chen et al. are truly associated with micro- or macroinvasion, they should independently predict adverse outcomes such as recurrence or shorter survival. In the absence of more clinically relevant outcome data, the conclusions of Chen and coworkers should be regarded as preliminary.

The study presents additional issues that highlight the need to consider its results preliminary. In $54 \%$ of their study population, cirrhosis was diagnosed based only on imaging without histopathology. It is possible that these $54 \%$ patients had more severe cirrhosis. In addition, the researchers diagnosed ascites based on ultrasonography and categorized the condition as "none," "mild-to-moderate," or "severe." In practice, it is difficult to detect ascites when the volume is less than 
$100 \mathrm{~mL}$, so some of the patients categorized as having "no" ascites in the study may have had mild ascites. This calls into question the researchers' recommendation for more frequent surveillance for patients with ascites. This and other conclusions in the study by Chen et al. require verification in larger studies that take a rigorous, systematic approach to clinical characterization and outcome analysis.

\section{References}

1. Torre LA, Bray F, Siegel RL, Ferlay J, Lortet-Tieulent J, Jemal A. Global cancer statistics, 2012. CA Cancer J Clin. 2015;65(2):87108 .

2. Forner A, Llovet JM, Bruix J. Hepatocellular carcinoma. Lancet. 2012;379(9822):1245-55.
3. Zhong JH, Ke Y, Wang YY, Li LQ. Liver resection for patients with hepatocellular carcinoma and macrovascular invasion, multiple tumours, or portal hypertension. Gut. 2015;64(3):520-1.

4. Zhong JH, Wu FX, Li H. Hepatic resection associated with good survival for selected patients with multinodular hepatocellular carcinoma. Tumour Biol. 2014;35(9):8355-8.

5. Zhong JH, Lu SD, Wang YY, Ma L, Li LQ. Intermediate-stage HCCupfront resection can be feasible. Nat Rev Clin Oncol. 2015;12(5): 295. doi:10.1038/nrclinonc.2014.122-c3.

6. Zhong JH, Rodriguez AC, Ke Y, Wang YY, Wang L, Li LQ. Hepatic resection as a safe and effective treatment for hepatocellular carcinoma involving a single large tumor, multiple tumors, or macrovascular invasion. Medicine (Baltimore). 2015;94(3), e396.

7. Zhong JH, Ke Y, Gong WF, et al. Hepatic resection associated with good survival for selected patients with intermediate and advancedstage hepatocellular carcinoma. Ann Surg. 2014;260(2):329-40.

8. Chen C, Chen DP, Gu YY, et al. Vascular invasion in hepatitis B virus-related hepatocellular carcinoma with underlying cirrhosis: possible associations with ascites and hepatitis B viral factors? Tumour Biol. 2015. doi:10.1007/s13277-015-3311-8. 